\title{
High-speed optical pulse train generation based on line-by-line spectral intensity coding
}

\author{
Zhang Jia, Kim Ik-Hwan, Hong Sang-Jeen and Seo Dong-Sun \\ Department of Electronics, Myongji University, San 38-2, Nandong, \\ Cheoin-gu, Yongin, Gyeongido 449-728, Korea \\ E-mail: sdsphoto@mju.ac.kr
}

Received: 10.02 .2011

\begin{abstract}
We have built a simple line-by-line spectral intensity coder with $4 \mathrm{GHz}$ resolution at $1.55 \mu \mathrm{m}$ using a grating, a collimating lens, and an intensity mask. By applying a $10 \mathrm{GHz}$ mode-locked spectral comb to the coder, we have generated relatively stable $20-100 \mathrm{GHz}$ pulse trains with $10 \mathrm{GHz}$ steps. The characteristics of generated pulse trains are examined quantitatively by measuring the corresponding optical spectra, time-domain waveforms, autocorrelation traces, and radio-frequency spectra.
\end{abstract}

Keywords: optical pulses, spectral intensity coding, repetition rate multiplication, pulse shaping

PACS: 42.65.Re, 42.79.Dj, 42.25.Fx

UDC: 535.243

\section{Introduction}

Optical processing based on individual spectral line control of a mode-locked laser, which is named as "line-by-line pulse shaping", is very attractive for arbitrary waveform generation, optical CDMA (code division multiple access), microwave photonics, etc. [1-3]. Simple examples of applications of the line-by-line pulse shaping are spectral intensity coding or filtering of low-speed mode-locked lasers for repetition rate multiplication. When compared with other repetition-rate multiplication methods, spectral filtering is inherently simple. We have earlier studied a simple spectral filtering of a mode-locked spectral comb using a high-finesse Fabry-Perot etalon filter and have successfully generated $40 \mathrm{GHz}$ and $100 \mathrm{GHz}$ pulse trains from a $10 \mathrm{GHz}$ mode-locked laser [4]. One of imaginable disadvantages of the etalon filtering method is a lack of flexibility. At the same time, the method to be suggested in the present work is simple like the spectral filtering scheme used in the study [4] and, in addition, reveals much greater flexibility.

A simple line-by-line spectral intensity coder built here is based on a reflective geometry [5].

Ukr. J. Phys. Opt. 2011, V12, №3 
For simplicity we have employed a single fibre-pigtailed collimator, removing an additional large beam expander used in Ref. [6], though we can still achieve high enough resolution for the line-by-line spectral coding of a $10 \mathrm{GHz}$ spectral comb. In addition, we have used a low-cost mask for spectral intensity coding, instead of expensive liquid-crystal modulator arrays used in the other works [5-7]. The two-dimensional pattern of the intensity mask has enabled us to select different sets of slits of which spacing is determined by the spectral position of a repetition-rate multiplied pulse train. In this way the scheme suggested can have reasonably good performance and more practical flexibility than the other spectral filtering configurations, e.g. the etalon filtering. Note that the scheme based on a fixed mask is much simpler than a similar scheme based on liquid-crystal modulator arrays [6].

\section{Experimental setup}

Our experiment setup is shown in Fig. 1. A $10 \mathrm{GHz}$ spectral comb generated from a mode-locked fibre laser is spectrally coded (for filtering) by a spectral line-by-line intensity coder. The coded output is amplified and measured by an optical spectrum analyser (a $0.07 \mathrm{~nm}$ resolution), an intensity autocorrelator, a $40 \mathrm{GHz}$ sampling scope, and a RF spectrum analyser (0-25 GHz) coupled with a $26 \mathrm{GHz}$ photodetector.

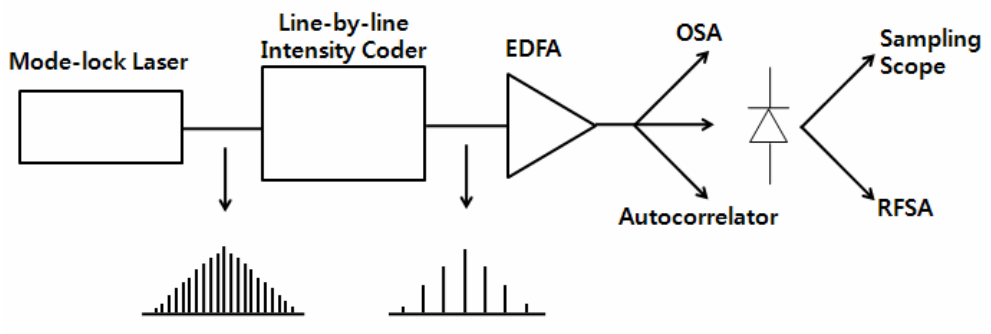

Fig. 1. Experiment setup (EDFA means erbium-doped fibre amplifier, OSA an optical spectrum analyser, and RFSA a radio-frequency spectrum analyser).

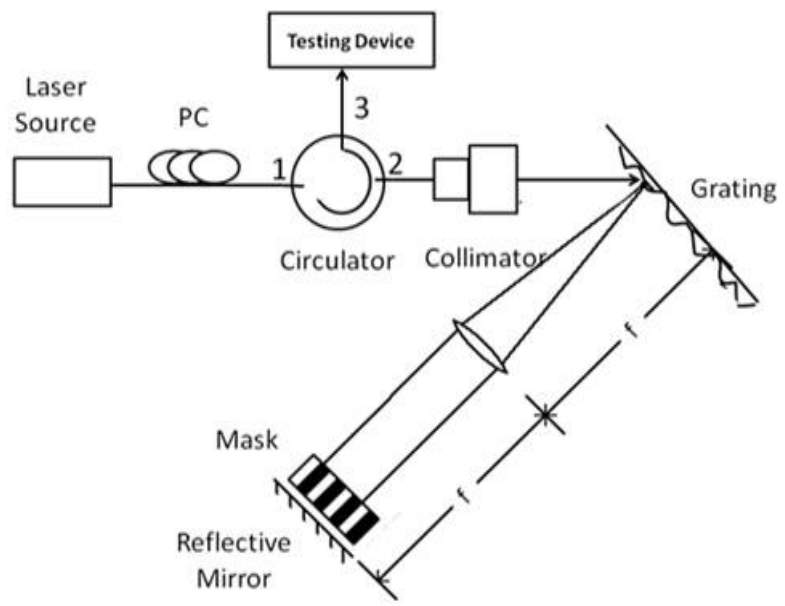

Fig. 2. Schematic diagram of our reflective line-by-line intensity coder ( $P C$ is a polarisation controller). 
Fig. 2 shows a schematic diagram of line-by-line intensity coder suggested by us, in which a fibre-coupled Fourier-transform pulse shaper is constructed in a reflective geometry. A fibre-pigtailed collimator expands the input beam to the size of $\sim 9 \mathrm{~mm}$ on a 1100 grooves $/ \mathrm{mm}$ grating, in order to enhance the coder resolution. Discrete spectral lines of a mode-locked laser are diffracted by the grating and focused by a lens with $50-\mathrm{mm}$ focal length. A polarisation controller ( $\mathrm{PC}$ in Fig. 2) is used to adjust for horizontal polarisation on the grating. A two-dimensional intensity mask of slits with different slit spacings is placed in the focal plane of lens to pass or block individual spectral lines. Details of split spacing and/or mask design will be discussed later.

Fig. 3 shows schematically a two-dimensional mask pattern and transmitted optical comb spectra corresponding to the individual slits. Here white bars represent the transparent slits and black parts correspond to the opaque regions. Due to Fourier-transforming properties of combination of the grating and the lens, the individual mode-locked spectral comb lines line up horizontally in a row of the mask. By moving the mask vertically, we can select a specific slit pattern for spectral intensity coding. For example, if the first row is selected, all of the spectral lines would pass through the mask. When the second column is selected, some other spectral lines would pass. This results in the spectral spacing of the passed signal becoming exactly twice the

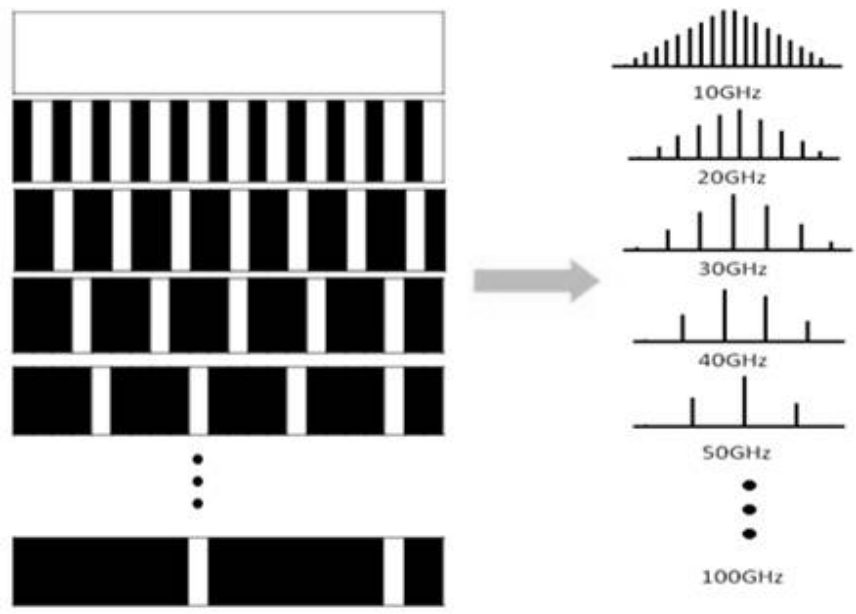

Fig. 3. Schemes of two-dimensional mask pattern (left) and transmitted optical comb spectra (right). input spacing. The latter means that the pulse repetition rate given by inverted spectral spacing is twice as large as the input one. In this way our simple mask enables line-by-line spectral intensity control over an entire optical band for various rates of repetition multiplication. A retro-reflecting mirror provides double-pass geometry and all of the spectral lines

transmitted through the mask are recombined into a single fibre and separated from the input via an optical circulator. The total loss inserted by an input circulator port 1 to an output port 3 is about $8.5 \mathrm{~dB}$. In view of the insertion loss, a weak signal should be amplified by an er-

Ukr. J. Phys. Opt. 2011, V12, №3 
bium-doped fibre amplifier (EDFA in Fig. 1) for easy measurements of its characteristics, such as autocorrelation traces.

Basing on the Fourier transforming property of the spectral coder shown in Fig. 2, one can get a relationship between the wavelength difference $(\Delta \lambda)$ and the spectral line spacing $(\Delta x)$ at the focal (or mask) plane for the first-order diffraction at the grating. Assuming that the horizontal direction at the mask plane is given by $x$ axis, one arrives at the following spectral slope $(\Delta \lambda / \Delta x)$ at the mask plane:

$$
\frac{\Delta \lambda}{\Delta x}=\frac{d \cos \theta_{d}}{f}
$$

where $d$ is the grating constant, $\theta_{d}$ the diffraction angle, and $f$ the focal length of the lens. For the grating of 1100 grooves $/ \mathrm{mm}$, the lens of $50 \mathrm{~mm}$ focal length, and the diffraction angle of $52^{\circ}$, the spacing between the $10 \mathrm{GHz}(0.08 \mathrm{~nm}$ at $1.55 \mu \mathrm{m})$ spectral lines at the mask plane becomes $72 \mu \mathrm{m}$. Based on this value, we can fix the width and/or the spacing of the mask pattern slits, as shown in Fig. 3.

\section{Spectral resolution and mask pattern design}

Now we obtain the beam radius $w_{o}$ at the mask plane,

$$
w_{0}=\frac{\lambda f \cos \theta_{i}}{\pi w_{i} \cos \theta_{d}},
$$

where $\lambda$ is the light wavelength, $\theta_{i}$ the angle of incidence at the grating, and $w_{i}$ the incident beam radius. We define the beam diameter, $2 w_{0}$, as a spectral resolution of the coder.

With our experimental parameters $\left(\lambda=1.55 \mu \mathrm{m}, w_{i}=9 \mathrm{~mm}, f=50 \mathrm{~cm}, \theta_{d}=52^{\circ}\right.$, and $\theta_{i}=66^{\circ}$ ) we get the beam diameter $2 w_{0}$ equal to $37 \mu \mathrm{m}$. From the relationship given by Eq. (1), we then obtain the spectral resolution of $5.2 \mathrm{GHz}$. Notice that the above resolution is still insufficient for controlling individual $10 \mathrm{GHz}$ spectral lines.

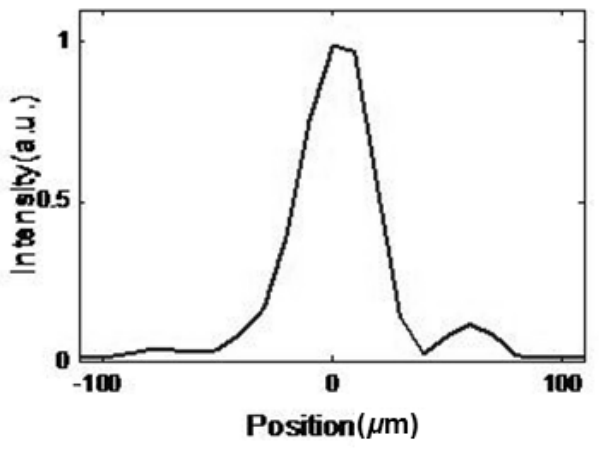

Fig. 4. Intensity distribution at the mask plane obtained for a monochromatic input of the coder.
In order to confirm the resolution and the spacing between 10 $\mathrm{GHz}$ spectral lines calculated here, a bare fibre tip coupled with an optical power meter has been placed at the mask plane (see Fig. 2). The output power coupled into the fibre tip has been recorded as a function of horizontal translation (along the $z$ axis). To find the 
spectral resolution, we have injected monochromatic light into the coder and measured the intensity distribution at the mask plane, as shown in Fig. 4. The beam width measured at a $1 / e^{2}$ power point is $\sim 50 \mu \mathrm{m}$, thus corresponding to the spectral resolution of $\sim 7 \mathrm{GHz}$. As we think, the beam width larger than its calculated value originates mainly from the resolution of the measuring system $(\sim 10 \mu \mathrm{m})$ and possible errors of the alignment.

Now we replace the monochromatic source by a $10 \mathrm{GHz}$ mode-locked comb source and perform similar measurements. Fig. 5 shows the results, which confirm that the spacing between the $10 \mathrm{GHz}$ spectral lines is $\sim 72 \mu \mathrm{m}$. This spacing agrees well with the calculated one. To check the accuracy of our system, the input optical spectrum measured by the optical spectrum analyser (Anritsu Model MS9710B; the resolution of $0.07 \mathrm{~nm}$ ) has been mapped onto the figure (red line). The both traces are well agreed in shapes, showing that the spectral comb lines are ready to be coded by a given mask pattern.

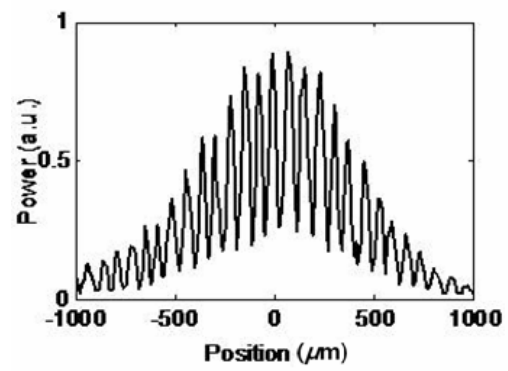

(a)

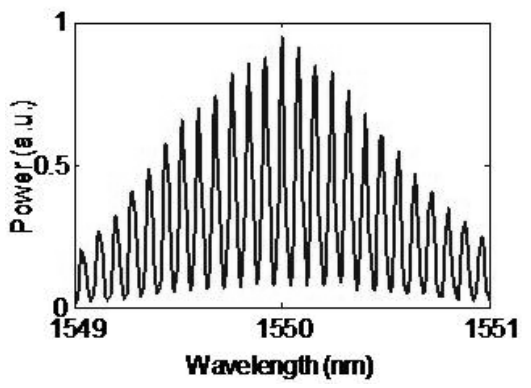

Fig. 5. Intensity distribution measured at the mask plane when a mode-locked comb source is applied at the coder input (a). For the sake of comparison, figure (b) shows the corresponding input spectrum measured by the optical spectrum analyser.

Basing on the experimental measurements, we can fix the detailed dimensions of the mask pattern shown schematically in Fig. 3. The width of $72 \mu \mathrm{m}$ for the transparent slits is sufficient to pass on almost all the power from a given spectral line and block any power from the unwanted spectral lines. The spacing between the slits is an integer multiple of $72 \mu \mathrm{m}$ (i.e., the spacing between the fundamental $10 \mathrm{GHz}$ spectral lines), as shown schematically in Fig. 3. Here the integer is determined by the pulse repetition rate factor intended. For instance, it becomes twice $72 \mu \mathrm{m}$ for the $20 \mathrm{GHz}$ train, three times larger than $72 \mu \mathrm{m}$ for the $30 \mathrm{GHz}$ train, and so on.

\section{Experimental results and discussions}

As we have already mentioned, a mode-locked laser output of $10 \mathrm{GHz}$ is characterised by evenly spaced discrete spectral lines (an optical frequency comb) with the frequency spacing equal to the pulse repetition rate at $10 \mathrm{GHz}$. The intensity mask in the Fourier-transform plane of the line-by-line intensity coder transmits the spectral lines among the $10 \mathrm{GHz}$ comb which are coincide with the transparent slits and it blocks (i.e., filters out) the unwanted spectral components

Ukr. J. Phys. Opt. 2011, V12, №3 
which fall into the opaque regions. This simple spectral filtering of the $10 \mathrm{GHz}$ spectral comb generates a new spectral comb with the spacing of an arbitrary integer multiple of $10 \mathrm{GHz}$. Even if careful adjustment for matching the spectral line positions and the mask slits is required, we can get reasonably good results (see some of them in Fig. 6). Fig. 6 proves successful generation of repetition-rate multiplied pulse trains (from $20 \mathrm{GHz}$ to $100 \mathrm{GHz}$, with a $10 \mathrm{GHz}$ step) using spectral filtering by the coder. A slight deformation of the transmitted spectrum and some residual power in the removed spectral components are observed due to mask-patterning errors and misalignment between the spectral comb and the mask slits. A similarity of the spectral envelopes observed on the input and output confirms that the individual pulse shapes remain almost same after the repetition rate multiplication.
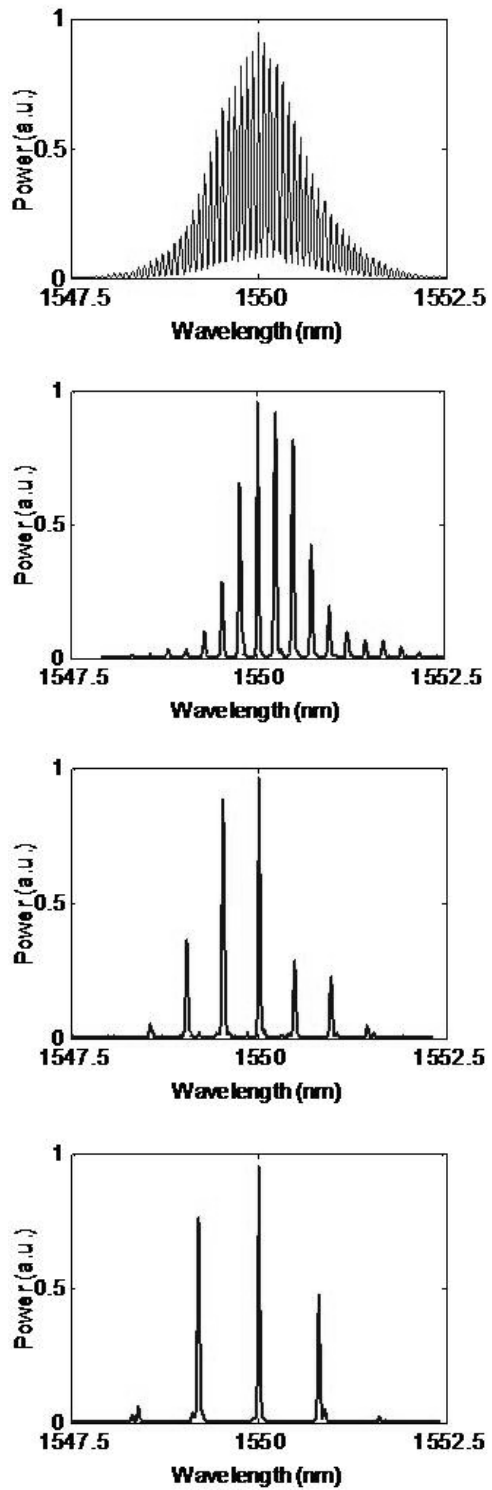

(a)
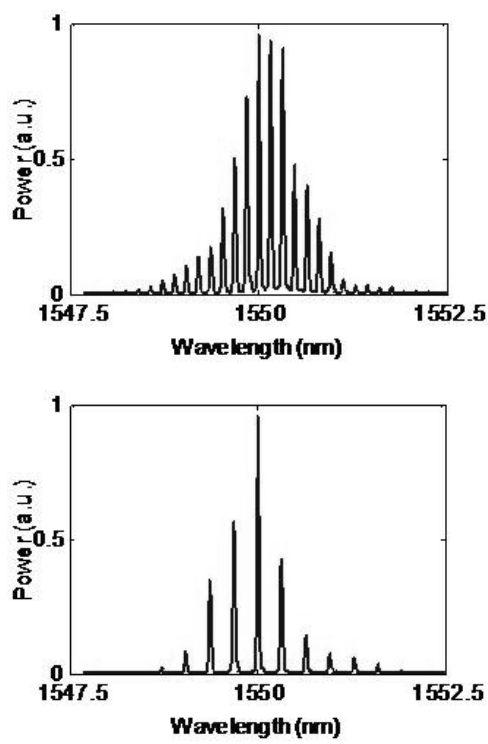

(d)

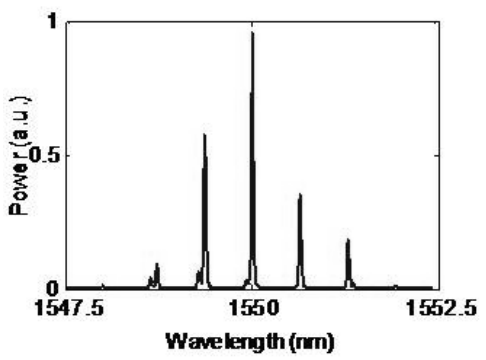

(f)

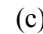

(e)

(g)

Fig. 6. $10 \mathrm{GHz}$ input (a) and filtered output optical spectra, showing repetition-rate multiplied pulse trains at $20 \mathrm{GHz}(\mathrm{b}), 30 \mathrm{GHz}(\mathrm{c}), 40 \mathrm{GHz}(\mathrm{d}), 60 \mathrm{GHz}(\mathrm{e})$, $80 \mathrm{GHz}(\mathrm{f})$, and $100 \mathrm{GHz}(\mathrm{g})$. 
To inspect quality of the pulse trains generated, we have measured the autocorrelation traces of the filtered outputs (see Fig. 7). As expected, the pulse period is reduced from 100 ps to 50 , $33.3,25,16.7,12.5$ and $10 \mathrm{ps}$, respectively. The traces also testify that both the input and output pulse widths are almost same, as expected for the spectra envelopes shown in Fig. 6. In this way we can reconfirm that the pulse trains from $20 \mathrm{GHz}$ to $100 \mathrm{GHz}$ are successfully generated from the $10 \mathrm{GHz}$ comb source, owing to simple spectral filtering based on our line-by-line spectral intensity coder.

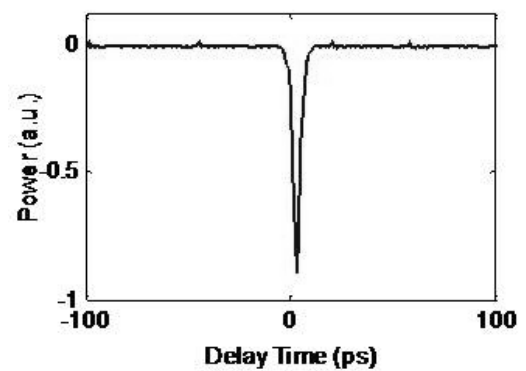

(a)
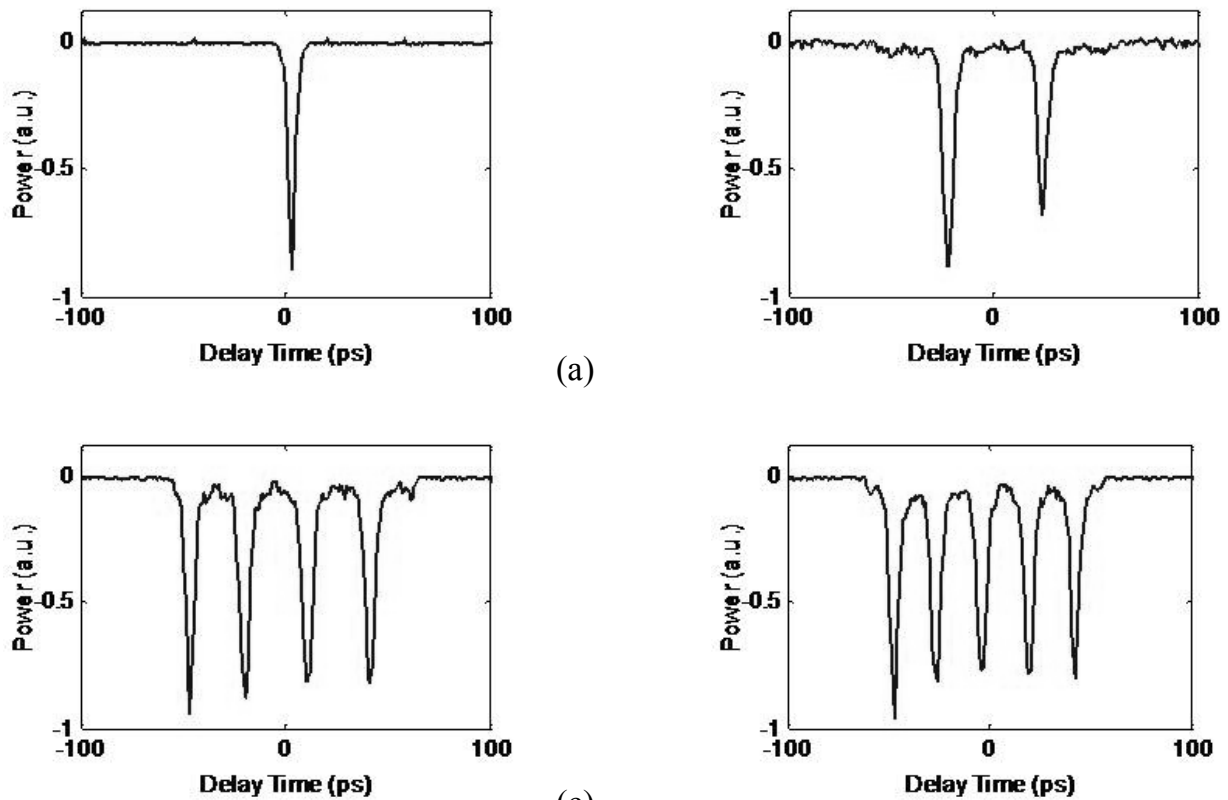

(b)

(c)

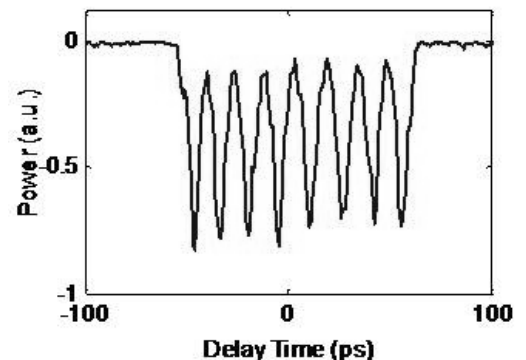

(e)

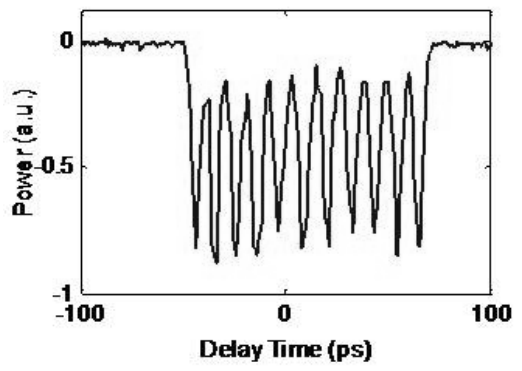

(d)

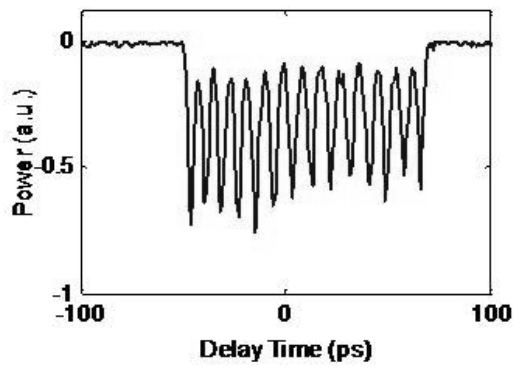

(g)

Fig. 7. Autocorrelation traces for (a) $10 \mathrm{GHz}$ (input), (b) $20 \mathrm{GHz}$, (c) $30 \mathrm{GHz}$, (d) $40 \mathrm{GHz}$, (e) $60 \mathrm{GHz}$, (f) $80 \mathrm{GHz}$, and (g) $100 \mathrm{GHz}$ trains.

Ukr. J. Phys. Opt. 2011, V12, №3 
Finally, we have measured directly the time traces for the $10 \mathrm{GHz}$ input and the outputs ranging from $20 \mathrm{GHz}$ to $40 \mathrm{GHz}$, using a $40 \mathrm{GHz}$ fast sampling scope (see Fig. 8). Again, reasonably stable 20, 30 and $40 \mathrm{GHz}$ pulse train generations are confirmed. We believe that a slight envelope modulation present at $10 \mathrm{GHz}$ comes from residual spectral components and a spectral envelope distortion shown in Fig. 5.
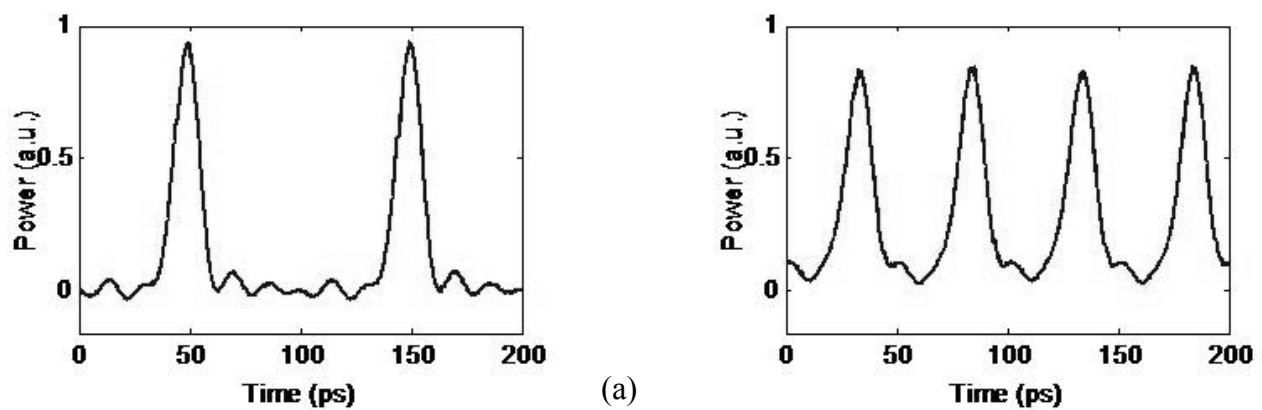

(b)

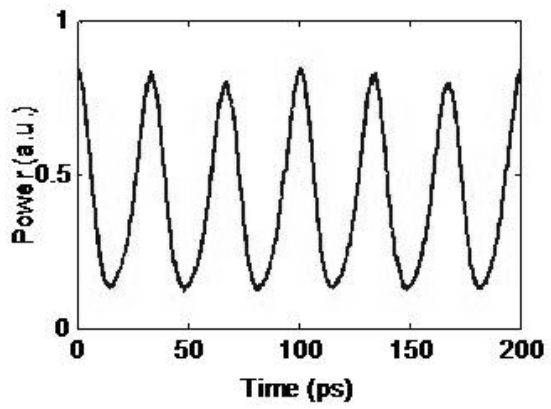

(c)

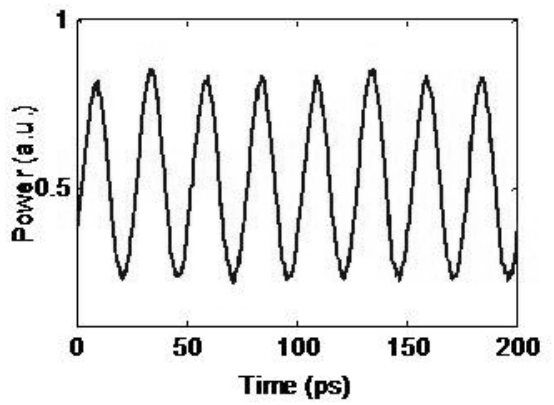

Fig. 8. Sampling scope traces for (a) $10 \mathrm{GHz}$ (input), (b) $20 \mathrm{GHz}$, (c) $30 \mathrm{GHz}$, and (d) $40 \mathrm{GHz}$ pulse trains.

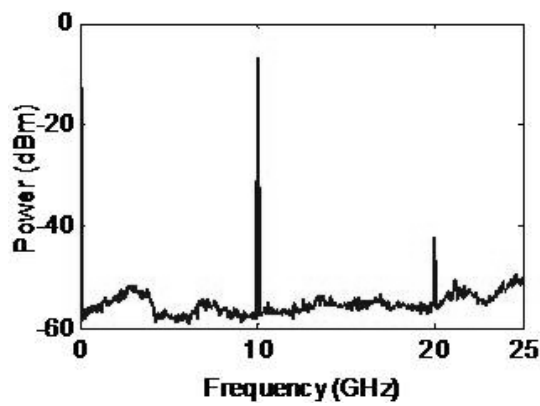

(a)
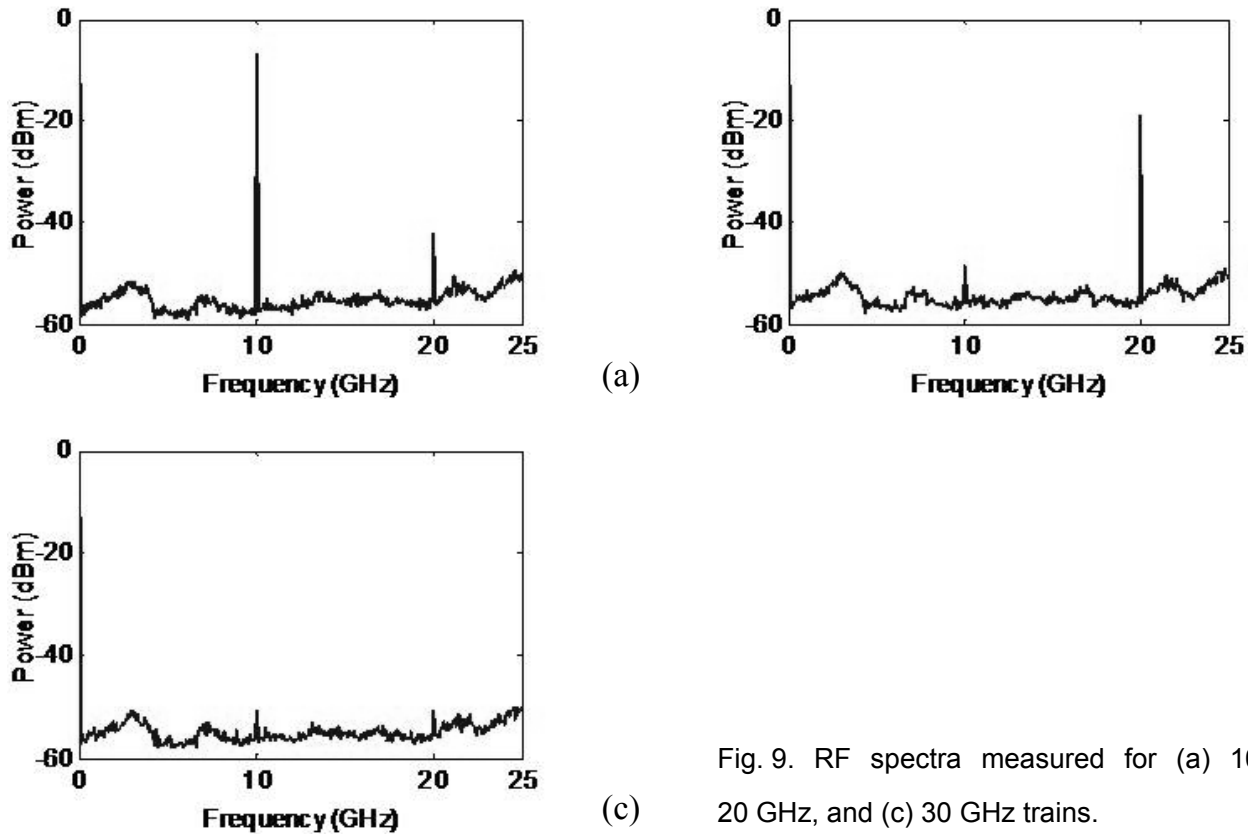

Fig. 9. RF spectra measured for (a) $10 \mathrm{GHz}$, (b) (c) $20 \mathrm{GHz}$, and (c) $30 \mathrm{GHz}$ trains. 
For checking the amount of the residual $10 \mathrm{GHz}$ component in the repetition-rate multiplied waveforms depicted in Fig. 8, we have detected the pulse trains with a fast detector and examined their radio-frequency spectra. For a comparison, we have kept almost the same optical power level at $\sim 0 \mathrm{dBm}$ for each waveform (Fig. 9). Here, the radio-frequency spectrum has been measured up to $25 \mathrm{GHz}$, the limit of our measurement facilities. The $10 \mathrm{GHz}$ input pulses show a clear $10 \mathrm{GHz}$ component and its second harmonic, as shown in Fig. 9a. For the $20 \mathrm{GHz}$ train, the $10 \mathrm{GHz}$ component is negligible ( $\sim 28 \mathrm{~dB}$ lower than the $20 \mathrm{GHz}$ signal - see Fig. 9b). The levels of suppression for the 10 and $20 \mathrm{GHz}$ components in the generated $30 \mathrm{GHz}$ pulse trains are similar (see Fig. 9c). The fact of sufficient suppression of the $10 \mathrm{GHz}$ component in both the 20 and $30 \mathrm{GHz}$ trains proves a stable repetition-rate multiplied pulse train generation.

\section{Conclusion}

We have built a simple and cost-effective line-by-line spectral intensity coder with the resolution of $\sim 7 \mathrm{GHz}$ at $1.55 \mu \mathrm{m}$. It utilises a grating, a collimating lens, an intensity mask, and a retro-reflection mirror. Basing on spectral filtering capability of this spectral intensity coder, we have experimentally generated multiple tens-of-GHz pulse trains at $20-100 \mathrm{GHz}$ (with the $10 \mathrm{GHz}$ step) from a mode-locked $10 \mathrm{GHz}$ laser. For spectral filtering, a simple and easily made intensity mask has been employed, which is placed in the Fourier-transform plane of our intensity coder. The quality of the high-speed pulse trains generated has been examined by measuring the optical spectra and the autocorrelation traces. Both the measurements have proved successful generation of the repetition-multiplied pulse trains, showing good suppression of the unwanted spectral lines and clear dips between the pulses seen in the autocorrelation traces. However, there has been a slight mismatch between the spectral line positions and the mask patterns that lead to some spectral envelope distortion and unwanted residual power of the transmitted signal spectra. The quality of the repetition-multiplied outputs has been examined in detail using the measurements of direct time traces and the corresponding radio-frequency spectra. Relatively clean sampling scope traces reconfirmed a fact of stable high-speed pulse train generation. The radio-frequency spectra have testified a suppression of $10 \mathrm{GHz}$ component greater than $28 \mathrm{~dB}$ for both the 20 and $30 \mathrm{GHz}$ pulse trains. It is also worthwhile that our technique is ready for applications when generating other high-speed pulse trains.

\section{Acknowledgment}

This work is supported by the Korea Research Foundation (\#2009-0072630).

\section{References}

1. Jiang Z, Leaird D E and Weiner A M, 2006. Optical processing based on spectral line-by-line pulse shaping on a phase-modulated CW laser. IEEE J. Quantum Electron. 42: 657-666. 
2. Kirchner M S and Diddams S A, 2010. Grism-based pulse shaper for line-by-line control of more than 600 optical frequency comb lines. Opt. Lett. 35: 3264-3266.

Fontaine N K, Scott R P, Cao J, Karalar A, Jiang W, Okamoto K, Heritage J P, Kolner B H and Yoo S J B, 2007. 32 phase $\times 32$ amplitude optical arbitrary waveform generation. Opt. Lett. 32: 865-867.

3. Zhang Jia, Hong Song-Jin and Seo Dong-Sun, 2010. High-speed pulse train generation by spectral filtering of a mode-locked laser output. Ukr. J. Phys. Opt. 11: 61-66.

4. Jiang Zhi, Huang Chen-Bin, Leaird D E and Weiner A M, 2007. Spectral line-by-line pulse shaping for optical arbitrary pulse train generation. J. Opt. Soc. Amer. B. 24: 2124-2128.

5. Thurston R N, Heritage J P, Weiner A M and Tomlinson W J, 1986. Analysis of picosecond pulse shape synthesis by spectral masking in a grating pulse compressor. IEEE J. Quantum Electron.

22: 682-696.

6. Caraquitena J, Jiang Z, Leaird D E and Weiner A M, 2007. Tunable pulse repetition-rate multiplication using phase-only line-by-line pulse shaping. Opt. Lett. 32: 716-718.

Zhang Jia, Kim Ik-Hwan, Hong Sang-Jeen and Seo Dong-Sun, 2011. High-speed optical pulse train generation based on line-by-line spectral intensity coding Ukr.J.Phys.Opt. 12: 117-126.

Анотація. У роботі запропоновано порівняно простий спектрально-лінійчатий кодер інтенсивності з розділенням 4 ГГц на довжині хвилі 1,55 мкм, у якому використано гратку, колімаційну лінзу та маску інтенсивності. Для гребінчастого спектру з періодом 10 ГГи і синхронізацією мод ми генерували доволі стабільний иуг імпульсів із кроком 10 ГГи і иириною 20 - 100 ГГи.. Характеристики генерованого иугу імпульсів досліджено шляхом вимірювання оптичних спектрів, форми сигналів, автокорелячійних слідів і радіочастотних спектрів. 